\title{
Note on Gauss' Proof of the Reciprocity of Parallelism.
}

\author{
By Dr D. M. Y. SommRrviLLE.
}

\section{(Read and Received 13th February 1914.)}

The proposition that if $\mathrm{AA}^{\prime} \| \mathrm{BB}^{\prime}$ then $\mathrm{BB}^{\prime} \| \mathrm{AA}^{\prime}$ appears at first sight so simple that it might be regarded as almost intuitive. This is because we already think of parallelism as a symmetrical relationship between two straight lines, in accordance with Euclid's definition of parallels as "straight lines which, being in the same plane and being produced indefinitely in both directions, do not meet one another in either direction." If we take along with this definition Euclid's fifth postulate, or Playfair's equivalent, it defines a unique line through a given point parallel to a given line; but, without the postulate, it cannot be assumed to define more than a class of lines, and a stricter definition is required. Gauss' definition is as follows :

The straight line $\mathrm{AA}^{\prime}$ is parallel to the straight line $\mathrm{BB}^{\prime}$ when

(1) the two lines lie in the same plane;

(2) $\mathrm{AA}^{\prime}$ does not cut $\mathrm{BB}^{\prime}$;

(3) any ray through $A$ within the angle $\mathrm{BAA}^{\prime}$ cuts $\mathrm{BB}^{\prime}$.

This definition does not involve the two lines symmetrically, and it is not so obvious that any ray through $B$ within the angle $\mathbf{A B B}^{\prime}$ cuts $\mathbf{A A}^{\prime}$, which is the reciprocal property that has to be proved.

It is possible to prove this by showing that a line can be drawn cutting $\mathrm{AA}^{\prime}$ at $P$ and $\mathrm{BB}^{\prime}$ at $Q$, making equal angles $\mathrm{A}^{\prime} P Q$ and $B^{\prime} Q P$, and then making use of the symmetry of the "corresponding points " $P, Q$. The object of this note, however, is not to establish the theorem in any new way, but to examine two proofs which Gauss has given for the theorem.

In Vol. 8 of Gauss' Works, pp. 202-209, there are printed three notes, undated, but supposed to have been written in the 
year 1831. No. 1 (pp. 202-206) is entitled "Parallellinien," and proves the three fundamental properties of parallelism:

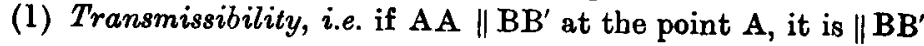
at any other point in its length;

(2) Reciprocity, i.e. if $a \| b$, then $b \| a$.

(3) Transitivity, i.e. if $a \| b$ and $b \| c$, then $a \| c$.

No. 2 (p. 207) deals with corresponding points, and No. 3 (pp. 208-209) is entitled "Parallelismus," and deals with the same matters as the other two, but only in outline.

No. 3 contains the following proof of the Reciprocity Theorem: "Let 1 and 2 be parallel. If now 2 is not parallel to 1 , let $c d$ " be parallel to 1. Let $c a$ be perpendicular to 1 and $a c b=a c b^{\prime}=\frac{1}{2} d c d^{\prime}$. Further $c b e=c b^{\prime} b$. Therefore be will cut 2, in $c^{\prime}$. Now make $b^{\prime} g=b e^{\prime}$, then $c g$ and $c d^{\prime}$ will make the same angle with $c b^{\prime}$. Which is absurd."

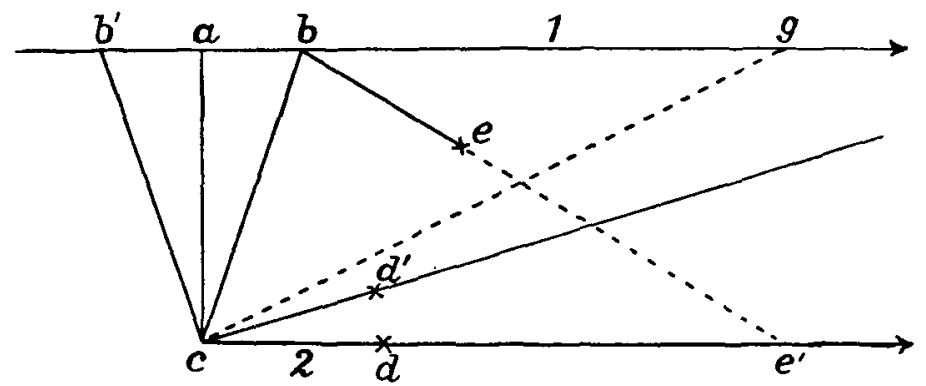

Fig. 1.

Now in this proof there is a fallacy, for it cannot be assumed, without assuming that $c d \| a b$, that the line $c b$ which makes with $c a$ an angle $=\frac{1}{2} d c d^{\prime}$ will meet 1 .

Bonola, in his book "La geometria non-euclidea," gives only a short resumé of Gauss' researches in the theory of parallels, and refers to this theorem in the single sentence, "this property [of reciprocity] forms the subject of another elegant demonstration of Gauss." Liebmann, in his German version of Bonola's book, changes this to "the following elegant demonstration of Gauss," and appends the above insufficient proof. The Russian translation follows Liebmann literally, but Professor Carslaw, in his English translation, has pointed out the fallacy, and gives instead the other proof by Gauss which is contained in No. 1 . 
This proof runs as follows* : "Let the straight line 1 be parallel to 2 . From any point $A$ in 2 let fall a perpendicular $A B$ on 1 . Let 3 be any straight line through $A$ between $A B$ and 2, and $A C$ a straight line between the same boundaries, so that the angle $\mathrm{BAC}=\frac{1}{2}(3,2)$.

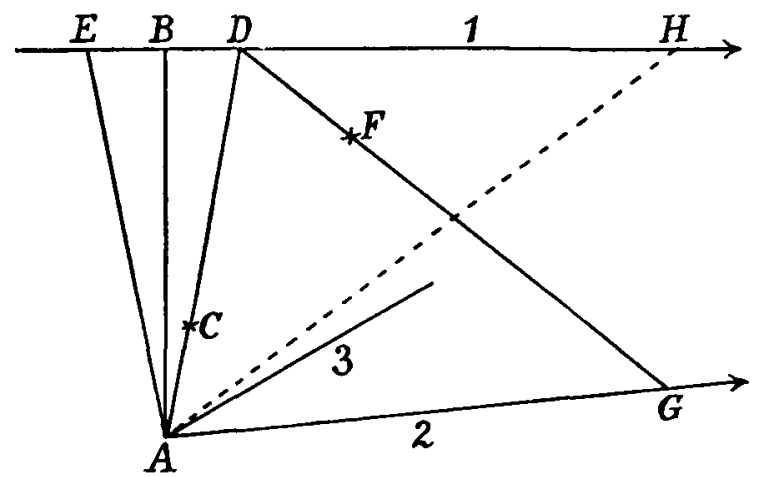

Fig. 2.

"We have now two cases to distinguish. (1) If AC...cuts the line 1 in $D$, make $B E=B D$, so that $E$ is taken on 1 on the opposite side from $D$. Through $D$ draw the straight line DF... between 1 and DA, so that $A D F=A E D$. This straight line will therefore cut 2 in $\mathrm{G}$. Make $\mathrm{EH}=\mathrm{DG}$ and join $\mathrm{AH}$. The triangles $\mathrm{ABD}, \mathrm{ABE}$ will be congruent, therefore $A E=A D$; consequently also the triangles ADG, $\mathbf{A E H}$ congruent, therefore $\mathrm{EAH}=\mathrm{DAG}$. $\mathrm{GAH}=\mathrm{DAE}=(2,3), \mathrm{AH}$ is identical with 3 , or 3 cuts 1 in $\mathrm{H}$, and consequently, since 3 may signify any line lying between 2 and $A B, 2$ is parallel to 1.

"(2) If $\mathrm{AC}$ does not cut 1 , let $\mathrm{D}$ be any point on 1 . There hold then the same conclusions as before up to the result $\mathrm{GAH}=\mathrm{DAE}$. Only in this case $\mathrm{DAB}<\mathrm{CAB}$ or $\mathrm{DAE}<(2,3)$. Therefore $(2,3)>\mathrm{GAH}$ and 3 will consequently lie in the closed figure $A H D$ and therefore cut $D H$. The rest as in (1)."

* Figs. 2 and 3 were added by the Editor. Note 1 was found apparently with no diagrams attached to it. 
It is interesting to find the "giant mathematician" at fault, though our triumph is short-lived when we find the deficiency so amply made good in the alternative proof.

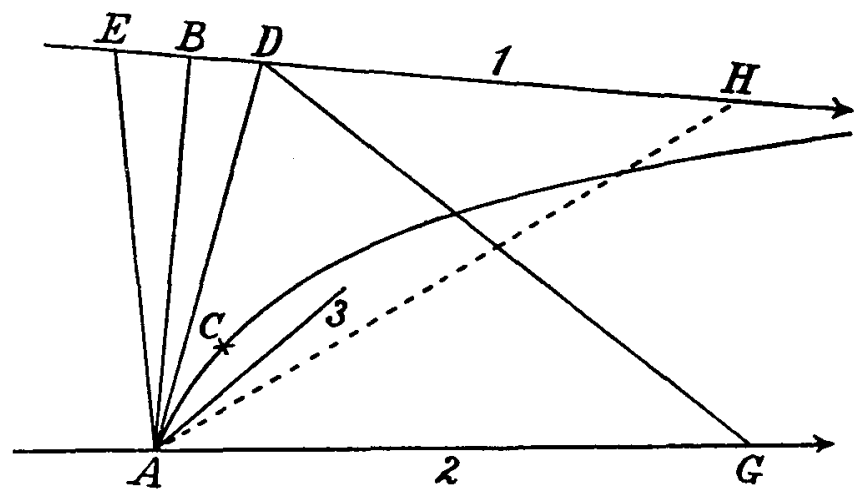

Fig. 3.

The occurrence of this fallacy proves almost conclusively, what is already indicated by the form of the three notes, ${ }^{*}$ that in chronological order No. 3 is the first. Its character suggests that it consisted of rough jottings and outlines of what Gauss works out more elaborately in Nos. 1 and 2. It is almost inconceivable that he should have altered, even in a synopsis, a perfectly valid proof into an invalid one simply for the sake of simplification. Prof. Stäckel, who edited the geometrical part of this volume of Gauss' Works, and to whom I communicated my idea of the chronological order of these notes, admits that there is undoubtedly much to be said for this view. The question will be considered by him in a complete report on Gauss' geometrical works which he is preparing for the Göttinger Nachrichten, as a contribution to a scientific biography of Gauss. Similar reports on Gauss' works in the theory of numbers and function-theory have already been published by Bachmann in 1911 and Schlesinger in 1912.

*For instance, in beginning the proof in No. 3 by the phrase, "Es sei 1 und 2 parallel," he implies the reciprocal relation that is to be proved. 